\title{
COVID-19, NUMÉRIQUE ET LIBERTÉS
}

\author{
Yves Poullet, Marie-des-Neiges Ruffo de Calabre, Jessica Lombard
}

\author{
S.E.R. | «Études»
}

2020/6 Juin | pages 57 à 66

ISSN 0014-1941

Article disponible en ligne à l'adresse :

https://www.cairn.info/revue-etudes-2020-6-page-57.htm

Distribution électronique Cairn.info pour S.E.R..

(c) S.E.R.. Tous droits réservés pour tous pays.

La reproduction ou représentation de cet article, notamment par photocopie, n'est autorisée que dans les limites des conditions générales d'utilisation du site ou, le cas échéant, des conditions générales de la licence souscrite par votre établissement. Toute autre reproduction ou représentation, en tout ou partie, sous quelque forme et de quelque manière que ce soit, est interdite sauf accord préalable et écrit de l'éditeur, en dehors des cas prévus par la législation en vigueur en France. Il est précisé que son stockage dans une base de données est également interdit. 


\section{Sociét é \\ COVID-19, \\ NUMÉRIQUE ET LIBERTÉS}

Yves POULLET, Marie-des-Neiges RUFFO de CALABRE et Jessica LOMBARD

Durant la pandémie, le numérique s'est révélé un outil précieux pour assurer une continuité de la vie professionnelle, de l'éducation, de la vie familiale et amicale, et assurer une appétence pour la vie culturelle disponible en ligne. La bataille contre le coronavirus grâce au numérique se joue également sur d'autres plans: des gouvernements, avec l'appui d'entreprises privées, développent de nouvelles politiques de surveillance de l'épidémie et des personnes infectées. Ces usages technologiques sont des gages d'efficacité mais également des facteurs de risques pour les libertés des citoyens.

Cet article, achevé le 10 avril, a été conçu avec la collaboration des membres du labo Ethics de l'Université catholique de Lille.

$\ll \bigcirc$ onfrontée à un virus inédit, la Chine a certainement déployé l'effort d'endiguement d'une maladie le plus ambitieux, agile et offensif de l'Histoire. $»^{1}$ Cette assertion provient du rapport de l'Organisation mondiale de la santé (OMS), en date du 28 février 2020. Il faut néanmoins souligner que, comme toute organisation, l'OMS n'est pas politiquement neutre.

1. Organisation mondiale de la santé (OMS), Report of the World Health Organization - China Joint Mission on Coronavirus Disease 2019 (Rapport de la mission conjointe Organisation mondiale de la santé et Chine sur la maladie à coronavirus 2019), 2020, p. 16. Pour une mise en perspective de l'absence de neutralité de l'OMS vis-à-vis de la Chine, voir Paul Benkimoun, "Coronavirus: comment la Chine a fait pression sur l'OMS ", Le Monde, 29 janvier 2020. 
Ce rapport semble indiquer ${ }^{2}$ que la situation que nous connaissons désormais dans le monde entier, et en France depuis la fin du mois de février, a été prise en charge en Chine avec succès. Il indique par ailleurs qu'une "grande partie de la communauté internationale n'est pas prête, ni mentalement ni matériellement, à mettre en place les mesures qui ont été employées par la Chine pour contenir le virus ». Si la communauté internationale n'est pas prête à gérer la pandémie de la même façon, il nous faut comprendre quelles sont ces mesures qui fonctionnent, mais qui questionnent la balance entre efficacité et libertés individuelles.

Le gouvernement chinois considère la Covid-19 comme la plus grave urgence sanitaire qui ait frappé le pays depuis la naissance de la République populaire de Chine en 1949. Les moyens mis en place pour gérer la pandémie sont impressionnants. Ils ont commencé par le confinement de Wuhan et de sa région, première zone à avoir été déclarée infectée, par la publication de consignes strictes de santé publique (prise de température, lavage des mains, port du masque, déjà largement répandu dans les agglomérations pour des raisons de pollution) et par la construction en un temps record d'hôpitaux de campagne. Le dépistage de la population contaminée s'est effectué par des systèmes de surveillance médicaux déjà existants. Il est à noter que ces systèmes ont reçu l'appui des comités de quartier dont les membres se chargent de surveiller, par des thermomètres à infrarouge, la température corporelle de leurs voisins, parfois sans leur accord.

Au-delà de l'exemple quotidien des thermomètres à infrarouge, la Chine fait usage de moyens technologiques très spécifiques pour limiter la taille des foyers de contamination. La surveillance de la population par la géolocalisation des smartphones a notamment servi à limiter les contacts de chacun, par le traçage des déplacements et des achats. Les données des applications et opérateurs mobiles (WeChat, Alipay...) permettent de suivre le circuit du détenteur d'un smartphone et de savoir s'il approche d'une zone à risque, d'un moyen de transport ou d'un individu déclaré porteur du virus. Dans plusieurs villes, des codes QR de couleur verte, orange ou rouge ont été individuellement distribués aux citoyens pour les autoriser ou non à utiliser certains moyens de transport ou à entrer dans certains quartiers, magasins ou restaurants. Grâce aux technologies de reconnaissance 
faciale, les contrevenants et les porteurs de virus peuvent être identifiés et leurs photos affichées dans les lieux publics.

La Chine a été le premier pays à devoir mettre des mesures en place face au virus. Ces mesures ont supposément eu un impact, lequel sera néanmoins à clarifier lors des prochains mois d'évolution de la maladie. Cet exemple nous invite donc à penser la teneur de la réponse faite à la pandémie en France. Le rapport de l'OMS rappelle que les mesures de surveillance mises en place par la Chine ne seraient probablement pas acceptées par la majorité des citoyens de nos démocraties occidentales.

Notre propos n'est pas de mettre au pilori l'action gouvernementale chinoise mais, à l'aune de cet exemple, de réfléchir sur la réponse à la pandémie, que nous devons construire ensemble en tenant compte des valeurs de notre démocratie. Dans cette lutte contre le virus, la dimension technologique des moyens utilisés doit être soulignée. Elle est à fois gage d'efficacité mais également facteur de risque.

\section{La tentation du numérique}

La pandémie rythme nos vies; la peur s'installe et nos maisons exiguës rendent le confinement difficilement supportable. Jamais le numérique n'est apparu aussi nécessaire: vidéoconférences professionnelles, familiales ou amicales; jeux, musique ou cinéma; achats; éducation en ligne... Si l'on peut espérer que le coronavirus aura perdu la partie, le numérique est en passe de gagner définitivement la bataille de la connexion de nos foyers et de nos entreprises. Mieux, le numérique nous promet de gagner la bataille contre l'épidémie.

Nonobstant le manque de recul nécessaire face à la maladie, les laboratoires scientifiques mettent en œuvre plusieurs outils d'intelligence artificielle pour chercher à comprendre le modus operandi de la maladie, détecter la qualité des solutions promises et pouvoir prédire les risques encourus. La bataille contre le coronavirus grâce au numérique s'opère également et surtout sur d'autres champs. Les gouvernements, souvent avec l'appui voire à l'initiative d'entreprises privées ${ }^{3}$,

3. Certaines firmes privées connaissent mieux l'évolution de la pandémie que nos gouvernements: Google et Facebook se sont ainsi proposé d'aider le gouvernement américain. En Belgique, on pointe l'initiative commune de trois opérateurs de mobilophonie, discutée et partiellement acceptée par le gouvernement belge. Voir également le projet d'Apple et de Google, décrit dans l'article de Russell Brandom et Adi Robertson, "Apple and Google are building a coronavirus tracking system into iOS and Android ", The Verge, 10 avril 2020, consultable sur www.theverge.com 
développent de nouveaux moyens de surveillance de l'épidémie, des personnes infectées et, en conséquence, de chacun de nous. Ils trouvent chez leurs citoyens, ayant la peur au ventre, des alliés des politiques de surveillance mises en place. Le drame des Twin Towers du 11 septembre 2001 n'est pas loin. Hier, le terrorisme; aujourd'hui, le coronavirus. L'angoisse que, par ailleurs, la rumeur portée par les réseaux sociaux ${ }^{4}$ amplifie est le meilleur allié de telles mesures politiques.

Dans nombre de pays, faute de masques en quantité suffisante et de tests pour détecter le coronavirus, les politiques trouvent des réponses certes dans le confinement mais également dans les technologies du numérique. La géolocalisation ${ }^{5}$ permet désormais (ce n'était pas le cas en 2001) de suivre chacun mais également de calculer la proximité physique avec son entourage. Un rapide tour du monde des technologies utilisées laisse apparaître des réponses variées, dont certaines paraissent préférables à d'autres. Les solutions chinoises rappelées ci-dessus se concentrent sur le traçage des individus, la reconnaissance faciale et le système d'alerte d'une personne suspectée d'être porteuse du coronavirus. Nombre de pays asiatiques (Corée du Sud, Vietnam, Taïwan) ont mis au point, à la suite d'un accord entre opérateurs de mobilophonie et gouvernements, des systèmes qui permettent de retracer hier et de suivre aujourd'hui les déplacements d'une personne atteinte de la maladie. D'autres pays - comme l'Italie et la Belgique - travaillent notamment sur des données agrégées et pseudonymisées, de manière à surveiller les déplacements des personnes, étudier les foyers d'épidémie et le respect de la distanciation sociale. D’autres solutions (Coronapp, application développée au niveau international, ou Covid19stop, en Belgique) proposent aux utilisateurs de renseigner leur état de santé et de partager leur géolocalisation. Elles reposent sur le volontariat des citoyens, appelés à stocker leurs données de déplacements et, grâce à un système Bluetooth, les téléphones situés à proximité. S'ils sont déclarés positifs au virus, ils sont invités à mettre ces données à disposition d'une autorité sanitaire, qui doit avertir les personnes les ayant côtoyés.

Ce tour d'horizon atteste d'une diversité de solutions. Le choix de l'une ou de l'autre est explicable tant par la possibilité pratique de

\footnotetext{
4. La lutte contre la désinformation sur le coronavirus est une préoccupation de la Commission européenne et de l'OMS. Voir https://ec.europa.eu/info/live-work-travel-eu/health/coronavirusresponse/fighting-disinformation_fr

5. Selon les spécialistes, I'utilisation de nouvelles fréquences (Galileo et GNSS [Global Navigation Satellite System]) permet d'arriver à une précision actuelle de trente centimètres.
} 
la mettre en place (le nombre de masques ou de tests), que par l'exigence d'efficacité qui nécessite de juguler la pandémie et par la culture d'un respect de l'autorité gouvernementale et de la collectivité propre à chaque nation. Chaque pays agit donc à partir des ressources dont il dispose et selon sa culture. La Chine n'a pas attendu la pandémie pour déployer des mesures de surveillance numérique à grande échelle de sa population et le mode de gestion choisi en cette crise ne peut surprendre. Mais que les pouvoirs publics des pays occidentaux choisissent des mesures qui semblent contraires à leurs habitudes en adoptant une surveillance massive peut en étonner plus d'un, même si, curieusement, nous l'acceptons dans les faits de la part des opérateurs de mobilophonie (par exemple, Google Now d'Androïd), des opérateurs de réseaux sociaux et autres plateformes. En réalité, l'usage par les autorités publiques de mesures de surveillance de grande ampleur (géolocalisation de la population, recours aux drones et aux caméras infrarouges) n'est pas inédit. En revanche, il a rarement été aussi explicite.

Par ailleurs, la société confucianiste chinoise pratique historiquement un contrôle social qui implique le respect de l'autorité en vue du bien commun. En France et ailleurs en Europe, la pratique de la délation pour non-respect du confinement existe et se confronte à la propension de certains à braver les interdits. Ainsi, les cultures maintiennent grandement les mœurs de chaque population, quand bien même les conventions sociales s'adaptent ${ }^{6}$. Bien entendu, certaines lois ne sont (heureusement) pas applicables partout, la crédibilité du pouvoir étatique reposant en partie sur sa capacité à les faire respecter et donc à les faire accepter à sa population.

\section{Quand la liberté se fait virtuelle}

Benjamin Franklin aurait affirmé « qu'un peuple prêt à sacrifier un peu de liberté pour un peu de sécurité ne mérite ni l'une ni l'autre, et finit par perdre les deux ». Pour paraphraser ce père fondateur d'une des premières démocraties modernes, demandons-nous si et comment le sacrifice indispensable et présent de nos libertés pour assurer une plus grande sécurité sanitaire peut encore préserver les deux?

6. Avant les mesures de distanciation sociale, les poignées de mains se sont transformées. Si le geste a évolué, l'habitus de se saluer demeure. 
En temps de pandémie, l'adoption des mesures de confinement imposées par la plupart des gouvernements limite la liberté de circulation, cette liberté si chère à Benjamin Constant « d'aller, de venir sans en obtenir la permission et sans rendre compte de ses motifs ou de ses démarches ${ }^{7}$. Même si la situation est exceptionnelle, l'enfermement pour des individus sains choque la théorie libérale. Elle s'explique à la lumière de théories libérales utilitaristes, telle celle de John Stuart Mill, pour qui la seule restriction étatique de nos libertés acceptables était précisément celle motivée par la sécurité de nos concitoyens: «La contrainte, exercée directement, ou sa répression par le biais de sanctions pénales, ne peut plus être admise comme un moyen de guider les hommes vers leur propre bien: elle se justifie uniquement dès lors qu'il s'agit de la sécurité des autres. ${ }^{8}$ La restriction des libertés des populations en situation de pandémie serait donc légitime dans la mesure où elle assure la sécurité d'autrui, et non pas pour assurer « leur propre bien».

Cependant, tandis que nous expérimentons ces restrictions et surveillances, comment pouvons-nous encore nous considérer comme des citoyens « libres » d'une démocratie plutôt que d'un régime autoritaire? Peut-être devons-nous compléter l'analyse de Constant sur la liberté des Anciens comparée à celle des Modernes, en découvrant un troisième âge de la liberté, celui de la pandémie de Covid-199. Aujourd'hui, la liberté ne serait ni celle de la participation politique des Anciens (en témoigne le taux d'absentéisme aux dernières élections françaises ${ }^{10}$ ), ni celle des Modernes (marquée par le repli sur ses intérêts privés). La « liberté en temps de pandémie » demeure certes marquée par ce repli sur la sphère privée, synonyme même de claustration domestique, mais est novatrice en ce que ces centres d'intérêt privés, pour être exercés, nécessitent le numérique, engendrant ainsi une forme nouvelle d'inégalité sociale.

Les victimes de la fracture numérique se découvrent les moins capables d'exercer une liberté en temps de pandémie, car elle est prin-

7. Benjamin Constant, De la liberté des Anciens comparée à celle des Modernes, 1819.

8. John Stuart Mill, De la liberté, 1859, Éditions John Parker et fils, p. 76. À noter qu'une page plus loin, le même auteur écrit: " Chacun est le gardien naturel de sa propre santé aussi bien physique que mentale et spirituelle."

9. Ceci compléterait la thèse de Benjamin Loveluck du libéralisme informationnel comme " la libre circulation de l'information (sous forme de code) comme principal socle de l'autonomie individuelle et collective ". Cf. B. Loveluck, Réseaux, libertés et contrôle. Une généalogie politique d'Internet, Armand Colin, 2015, p. 20.

10. La Covid-19 a accru ce désintérêt de la chose publique, chacun ayant préféré sa sécurité sanitaire. 
cipalement « virtuelle ». Avec la pandémie, on découvre que l'accès au numérique est une condition indispensable pour demeurer un citoyen, avoir accès à la culture, à l'éducation, ou simplement imprimer un formulaire de sortie. Si un déconfinement progressif devait être monitoré par nos données mobiles, les antismartphones seraient-ils ostracisés? Pour ne pas avoir cédé à un Apple ou un Samsung par souci de leur vie privée, seront-ils privés de mobilité? Sans compter que l'accès au numérique n'est jamais définitivement acquis. Outre sa vulnérabilité en cas de panne imprévue, il se révèle périssable dans le temps et nécessite un réinvestissement financier régulier. D’une part, l'obsolescence programmée, de l'autre, le manque de moyens financiers pour s'offrir un portable dernier cri condamnent à plus ou moins long terme l'utilité d'un objet technique dès lors qu'une mise à jour du système ne «supportera plus » les modèles plus anciens.

\section{Une facture par trop lourde?}

Si la liberté de mouvement se paie au prix de la vie privée en temps de pandémie, assurons-nous que la facture ne soit pas trop lourde et, au-delà, ne devienne un impôt perpétuel. Peut-on croire à un pays démocratique assurant, d'une part, la liberté de circuler et de décider de sa santé, et la sécurité sanitaire en temps de pandémie, d'autre part? Dans un article paru dans le Financial Times ${ }^{11}$, Yuval Noah Harari présentait que liberté et sécurité ne devraient jamais s'exclure. La Corée du Sud semble ainsi un exemple à méditer. Sans confinement, l'épidémie est pourtant sous contrôle. En plus des mesures de quarantaine ciblées et de larges dépistages, le numérique semble ainsi avoir assuré liberté de mouvement et sécurité sanitaire, par le backtracking des données téléphoniques. Cependant, selon l'article publié par l'institut Montaigne à propos de cet exemple coréen, «l'atteinte à la vie privée devient si forte que la Commission nationale [coréenne] des droits de l'homme s'inquiète de la divulgation excessive d'informations privées sur les patients, trop aisément identifiables par leur lieu de travail ou leurs antécédents de voyage $»^{12}$.

11. Yuval Noah Harari, "Le monde dans lequel nous vivrons, après l'orage " (traduction), L'Express, 26 mars 2020, consultable sur www.lexpress.fr

12. "Corée du Sud: dépistages, investigations ciblées et la question de la vie privée ", dans Mathieu Duchâtel, François Godement et Viviana Zhu, Coronavirus: l'Asie orientale face à la pandémie, Institut Montaigne, avril 2020, consultable sur www.institutmontaigne.org 
Quelles conséquences en tirer? Notre propos n'est certes pas de condamner l'utilisation des technologies, ni d'en privilégier une, mais de réclamer, avec d'autres, que nos autorités opèrent un juste équilibre entre nos libertés individuelles, d'une part, et l'intérêt général ou celui d'autrui, d'autre part. Sans doute est-il utile d'opérer cette balance à l'aune des circonstances actuelles et des risques particuliers et majeurs liés à la pandémie et de ne pas figer les équilibres habituels, du moins de manière temporaire ${ }^{13}$ ? Affirmer les libertés individuelles sans prendre en compte l'impact de nos comportements sur autrui et sur l'intérêt général économique, social et démocratique n'aurait pas de sens. La vie privée est un autre mot pour le développement de nos capacités dans une société donnée; elle doit prendre nécessairement en considération que l'homme est un être social, qu'il porte en soi tant le souci de lui-même que de l'autre. La dimension sociale de l'individu justifie la restriction des libertés, celle de circulation, celle de regroupement, mais il importe que ces restrictions, même ordonnées à la hâte, trouvent un fondement légal, soient motivées et proportionnées et surtout soient l'objet d'un minimum de consensus bien au-delà des seuls experts, même si ceux-ci ont un rôle clé dans l'éclairage que leurs réponses peuvent donner aux multiples interrogations du public. Comme l'écrit le Comité consultatif national d'éthique (CCNE) consulté par le gouvernement français ${ }^{14}$ : "L’un des enjeux éthiques majeurs dans cette situation, avec le passage à la dimension épidémique et collective, est d'engager la société tout entière dans une véritable démarche de responsabilité et de solidarité, parce que la lutte contre une épidémie doit être l'affaire de tous et pas seulement l'affaire des experts et des professionnels de santé. »

Le confinement n'aura, espérons-le, qu'un temps. Il y aura un " après ». Nos démocraties survivront-elles à la pandémie? Les mesures " d'exception ", "d'urgence ", " d'état d'urgence sanitaire » ou de " guerre » ne vont-elles pas plutôt se sédimenter en des mesures pérennes? Faut-il en effet rappeler que les mesures prises en sep-

\footnotetext{
13. "Lorsque des restrictions sont appliquées, ces mesures doivent être prises uniquement à titre provisoire et uniquement pour une période expressément limitée à l'état d'urgence. Il est également crucial que des garanties spécifiques soient mises en place et que des assurances soient données que les informations personnelles seront pleinement protégées, une fois l'état d'urgence levé. " D'après le Conseil de l'Europe, Joint statement on the right to data protection in the context of the Covid-19 pandemic (Déclaration commune sur le droit à la protection des données dans le contexte de la pandémie de Covid-19), 30 mars 2020, consultable sur www.coe.int/dataprotection

14. Contribution du Comité consultatif national d'éthique (CCNE), Enjeux éthiques face à une pandémie, 13 mars 2020.
} 
tembre 2001 sont toujours bien présentes sans que leur efficacité n'ait été démontrée, loin de là, et sans que leur légitimité n'ait été réévaluée? En France, le fondement légal du confinement s'inspire en effet de deux origines militaires, faisant de la référence à la guerre plus qu'une métaphore. Le premier décret du 16 mars 2020 en appelait à la théorie des circonstances exceptionnelles, remontant à la Première Guerre mondiale; la loi du 23 mars 2020, d'une autre votée au début de la guerre d'Algérie, en 1955. Actons à regret que cette loi du 23 mars, inscrivant " l'état d'urgence sanitaire » dans le Code de la santé fait désormais partie de «l'arsenal » juridique de l'État pour longtemps ${ }^{15}$. Enfin, si l'autorité publique peut en la matière entendre raison, qui dira demain aux " géants du Net ", dès le lendemain de la pandémie, de ne plus exploiter les innovations développées « en temps de guerre »?

\section{Pour une solidarité "pandémique "}

La pandémie met en lumière moins l'emploi du numérique, en tant que tel, que ce que son emploi sous-tend implicitement comme conception de l'homme. En temps de "guerre sanitaire ", l'individu est considéré par défaut comme dangereux, comme un facteur de contamination, qu'autrui est invité à suspecter voire à dénoncer. Intelligence artificielle aidant, il s'agit de le suivre voire de prévoir son comportement et d'empêcher son passage à l'acte. Tout cela au nom $\mathrm{du}$ « bien commun ». La métaphore de la guerre employée par le président Emmanuel Macron laisse sous-entendre que l'état d'exception justifierait n'importe quelle décision étatique. Or, tout état de guerre est encadré par des règles, notamment celle de la proportionnalité. Le maintien de nos libertés, même « en temps de guerre » contre la pandémie, doit être jugé proportionnellement à la menace et évoluer avec elle. Le numérique, par les possibilités de surveillance ubiquitaire et instantanée qu'il offre, devient l'instrument sans failles d'exercice du contrôle des mesures décrétées, un instrument dès lors tentant pour l'État, appuyé par le discours d'entreprises privées qui y trouvent une occasion de légitimer et de tester leurs innovations.

La dimension globale de la pandémie rejoint celle du numérique. La maladie nous embarque tous dans une aventure commune. Nous

15. Franck Johannes, "Coronavirus: l'état d'urgence en France bouscule l'État de droit ", Le Monde, 30 mars 2020, sur www.lemonde.fr 
sommes de fait tous solidaires. Les États savent que la victoire finale ne peut dépendre d'eux seuls. Pourtant, il est patent que les réponses et, en particulier, celles provenant de l'utilisation du numérique sont nationales et oublient la solidarité européenne. Si nous ne plaidons pas pour une réponse mondiale, faute d'acteurs capables de la définir, il est indispensable que les solutions nationales, si elles responsabilisent les acteurs locaux, ne conduisent à un repli sur nos « souverainetés ", excluant la préoccupation des populations les plus démunies face à la pandémie. Nous plaidons pour un dialogue, une prise en considération de l'apport que chaque solution peut apporter à la communauté mondiale. Pari à relever, dès aujourd'hui, au niveau européen; demain, au niveau mondial. L'organisation de ce dialogue suppose le rappel des valeurs éthiques universelles mises en évidence par l'Unesco: la dignité, celle d'un homme non épié continuellement; l'autonomie qui implique de responsabiliser son propre comportement à l'aune des impacts qu'il peut avoir pour autrui; la justice sociale qui nous rappelle l'inégalité des chances face à la pandémie (les personnes âgées dans les maisons de retraite, les personnes en situation de handicap, les personnes aux revenus précaires, les prisonniers) et vis-à-vis de l'accès au numérique... et donc des libertés encore accessibles demain. Il importe surtout de souligner, avec le premier article de notre loi "Informatique et libertés ", que la technologie est au service du développement humain et ne peut être l'outil d'une démagogie d'autant plus triomphante qu'elle est fondée sur la peur.

Faute de rechercher un tel dialogue où des voix multiples doivent pouvoir se faire entendre, il est à craindre qu'au lendemain de la crise sanitaire que nous traversons, nous nous réveillions plus désunis et moins humains que jamais, incapables de comprendre pourquoi nous n'avons pu faire de cette crise l'apprentissage d'un vouloir vivre commun et solidaire.

Yves POULLET, Marie-des-Neiges RUFFO de CALABRE et Jessica LOMBARD 